\title{
Scoring Methods of Polysomnography for Diagnosis of Sleep Apnea in Adolescents
}

\author{
Keu Sung Lee ${ }^{1}$, Seung Soo Sheen ${ }^{1}$, Il Jae Lee ${ }^{2}$, Byung-Joo Choi ${ }^{3}$, \\ Ji Ho Choi ${ }^{4}$, Do-Yang Park ${ }^{5}$, Han Tai Kim ${ }^{5}$, and Hyun Jun Kim ${ }^{5}$ \\ ${ }^{1}$ Departments of Pulmonary and Critical Care Medicine, ${ }^{2}$ Plastic and Reconstructive Surgery, ${ }^{3}$ Cardiology, ${ }^{5}$ Otolaryngology, \\ Ajou University School of Medicine, Suwon; and ${ }^{4}$ Department of Otorhinolaryngology-Head and Neck Surgery, \\ Soonchunhyang University College of Medicine, Bucheon Hospital, Bucheon, Korea
}

\author{
청소년에서 수면 무호흡 진단을 위한 수면 다원 검사의 판독 방법 \\ 이규성 ${ }^{1} \cdot$ 신승수 $^{1} \cdot$ 이일재 $^{2} \cdot$ 최병주 $^{3} \cdot$ 최지호 $^{4} \cdot$ 박도양 $^{5} \cdot$ 김한태 $^{5} \cdot$ 김현준 $^{5}$ \\ 아주대학교 의과대학 호흡기내과학교실, ${ }^{1}$ 성형외과학교실, ${ }^{2}$ 순환기내과학교실, ${ }^{3}$ 이비인후과학교실, ${ }^{5}$ \\ 순천향대학교 의과대학 부천병원 이비인후과학교실 ${ }^{4}$
}

\author{
Received January 30, 2018 \\ Revised April 18, 2018 \\ Accepted April 25, 2018 \\ Address for correspondence \\ Hyun Jun Kim, MD, PhD \\ Department of Otolaryngology, \\ Ajou University School of Medicine, \\ 206 Worldcup-ro, Yeongtong-gu, \\ Suwon 16499, Korea \\ Tel $+82-31-219-5267$ \\ Fax $+82-31-219-5264$ \\ E-mail entkhj@ajou.ac.kr
}

\begin{abstract}
Background and Objectives Respiratory scoring guidelines for children and adults have been used for evaluating adolescents both in the 2007 and 2012 American Academy of Sleep Medicine (AASM) scoring manuals. We compared the scoring methods of polysomnography used in these scoring manuals, where pediatric and adult scoring rules were adopted for the diagnosis of sleep apnea in adolescents.

Subjects and Method 106 Korean subjects aged between 13 and 18 years were enrolled. All subjects underwent overnight polysomnography in a sleep laboratory. Data were scored according to both pediatric and adult guidelines in the 2007 and 2012 AASM scoring manuals.

Results Both pediatric and adult apnea hypopnea index (AHI) using the 2012 method were significantly higher than those using the 2007 method. The difference in AHI compared between pediatric and adult scores with the 2012 AASM scoring system was markedly decreased from that with the 2007 method. There was a significant discordance in sleep apnea diagnosis between pediatric and adult scoring rules in the 2012 method.

Conclusion Both pediatric and adult rules were used for the diagnosis of adolescent sleep apnea in the 2012 method. However, there was significant discordance in the diagnosis between pediatric and adult scoring guidelines in the 2012 AASM manual, probably due to different cut-off values of AHI for the diagnosis of sleep apnea in pediatric $(\geq 1)$ and adult $(\geq 5)$ patients. Further studies are needed to determine a more reasonable cut-off value for the diagnosis of sleep apnea in adolescents. Korean J Otorhinolaryngol-Head Neck Surg 2018;61(11):593-9
\end{abstract}

Key Words Adolescent · Polysomnography · Scoring · Sleep apnea.

\section{Introduction}

Obstructive sleep apnea (OSA) is a sleep breathing disorder characterized by prolonged partial upper airway obstruc-

This is an Open Access article distributed under the terms of the Creative Commons Attribution Non-Commercial License (https://creativecommons.org/licenses/by-nc/4.0) which permits unrestricted non-commercial use, distribution, and reproduction in any medium, provided the original work is properly cited. tion or intermittent complete obstruction that disrupts normal ventilation. ${ }^{1)}$ Sleep apnea is a relatively common disorder in children with a prevalence of $1 \%$ to $5 \% .{ }^{2)}$ It is the most common pediatric sleep disorder, which is associated with serious neurocognitive, behavioral, growth, and cardiovascular abnormalities in children. ${ }^{3,4)}$ The pathophysiology of childhood sleep apnea has yet to be elucidated. 
Overnight polysomnography (PSG) in a sleep laboratory is the gold standard diagnostic method for childhood sleep apnea. $^{5-7)}$ Adolescence is a period of transition from childhood to adulthood with dynamic physical, developmental, and psychological changes. ${ }^{8)}$ No specific scoring method is available for PSG-based diagnosis of sleep apnea in adolescents. The American Academy of Sleep Medicine (AASM) published the AASM manual for the evaluation of sleep and associated events in 2007. ${ }^{6)}$ The 2007 manual recommended that respiratory scoring rules for children applicable for children under 18 years. It also recommended that an individual sleep specialist may prefer adult criteria to score children aged above 13 years. ${ }^{6}$

A few studies have reported that apnea-hypopnea index (AHI) is higher in adolescents when using the pediatric rules compared with the adult rules under the 2007 system. ${ }^{9)}$ The revised 2012 AASM scoring manual suggested fewer differences in AHI for adolescents when using adult rather than pediatric rules because of similarities between adult and pediatric hypopnea in the revised scoring manual. ${ }^{10)}$ Studies evaluating the differences between 2007 and 2012 methods for diagnosis of sleep apnea in adolescents have yet to be published. Therefore, the objective of this study was to evaluate the differences in scoring with overnight PSG using both 2007 and 2012 AASM scoring manuals comparing the effi- cacy of pediatric and adult scoring rules for the diagnosis of sleep apnea in adolescents.

\section{Subjects and Method}

\section{Subjects}

A total of 106 Korean subjects aged between 13 and 18 years and suspected of sleep disorders were enrolled in this study. For evaluation of sleep apnea, all subjects underwent overnight PSG in a sleep laboratory at Ajou University Hospital from Jan 2011 to Jan 2015. Anthropometric parameters including height and weight were measured in all study subjects at the time of PSG. Body mass index was calculated as kilograms per square meter. Subjects with prior surgical history of upper airway, congenital anomalies of face and upper airway, narcolepsy, and neuropsychiatric disorders were excluded from this study. This study was approved by the Institutional Review Board of Ajou University Hospital (IRB No. AIRB-MED-MDB-15-006). Informed consent was waived because this study was a retrospective study without interventions.

\section{Overnight polysomnography}

All participants were examined with an Embla N7000 system (Embla systems, Broomfield, CO, USA) in a sleep labo-

Table 1. Summaries for pediatric and adult rules in 2007 and 2012 AASM scoring manuals for diagnosing OSA

\begin{tabular}{|c|c|c|}
\hline & Pediatric rule & Adult rule \\
\hline \multicolumn{3}{|c|}{2007 AASM scoring manual } \\
\hline Apnea & $\begin{array}{l}\text { Peak signal excursions drop by } \geq 90 \% \text { of pre-event } \\
\text { baseline } \\
\text { Duration; } \geq 2 \text { missed breaths } \\
\geq 90 \% \text { of duration meets amplitude criteria }\end{array}$ & $\begin{array}{l}\text { Peak signal excursions drop by } \geq 90 \% \text { of pre-event } \\
\text { baseline } \\
\text { Duration; } \geq 10 \text { seconds } \\
\geq 90 \% \text { of duration meets amplitude criteria }\end{array}$ \\
\hline Hypopnea & $\begin{array}{l}\text { Peak signal excursions drop by } \geq 50 \% \text { of pre-event } \\
\text { baseline } \\
\text { Duration; } \geq 2 \text { missed breaths } \\
\geq 3 \% \text { desaturation from pre-event baseline or an } \\
\text { arousal } \\
\geq 90 \% \text { of duration meets amplitude criteria }\end{array}$ & $\begin{array}{l}\text { Rule } A \text {; } \\
\text { Peak signal excursions drop by } \geq 30 \% \text { of pre-event } \\
\text { baseline } \\
\text { Duration; } \geq 10 \text { seconds } \\
\geq 4 \% \text { desaturation from pre-event baseline } \\
\geq 90 \% \text { of duration meets amplitude criteria }\end{array}$ \\
\hline \multicolumn{3}{|c|}{2012 AASM scoring manual } \\
\hline Apnea & $\begin{array}{l}\text { Peak signal excursions drop by } \geq 90 \% \text { of pre-event } \\
\text { baseline } \\
\text { Duration; } \geq 2 \text { missed breaths }\end{array}$ & $\begin{array}{l}\text { Peak signal excursions drop by } \geq 90 \% \text { of pre-event } \\
\text { baseline } \\
\text { Duration; } \geq 10 \text { seconds }\end{array}$ \\
\hline Hypopnea & $\begin{array}{l}\text { Peak signal excursions drop by } \geq 30 \% \text { of pre-events } \\
\text { baseline } \\
\text { Duration; } \geq 2 \text { missed breaths } \\
\geq 3 \% \text { Desaturation from pre-event baseline or an } \\
\text { arousal }\end{array}$ & $\begin{array}{l}\text { Peak signal excursions drop by } \geq 30 \% \text { of pre-event } \\
\text { baseline } \\
\text { Duration; } \geq 10 \text { seconds } \\
\geq 3 \% \text { desaturation from pre-event baseline or an } \\
\text { arousal }\end{array}$ \\
\hline
\end{tabular}

AASM: American Academy of Sleep Medicine, OSA: obstructive sleep apnea 
ratory at Ajou University Hospital. The PSG recording channels comprised 4-channel electroencephalogram, 2-channel electro-oculogram, 1-channel submental electromyogram, 1-channel electrocardiogram, 1-channel tibial electromyogram, pulse oximetry, nasal cannula, oronasal thermistor, thoracic and abdominal respiratory effort bands, and bodyposition sensors. During the study, a technician monitored sleep activity on a video monitor and a computer screen that displayed all real-time data. One specialist manually scored all the PSG data with the 2007 and 2012 AASM scoring rules. Another specialist manually scored all the data again

Table 2. Characteristics of study participants

\begin{tabular}{lc}
\hline \multicolumn{1}{c}{ Characteristic } & Data \\
\hline Number of subjects & 106 \\
Sex (male:female) & $75: 31$ \\
Age (year) & $14.8 \pm 1.4$ \\
Body mass index $\left(\mathrm{kg} / \mathrm{m}^{2}\right)$ & $22.6 \pm 4.1$ \\
Respiratory rates during stage N2 (breaths/min) & $15.7 \pm 2.1$ \\
\hline
\end{tabular}

Data were presented as mean \pm standard deviation or number. N2: stage 2 sleep

Table 3. Sleep architecture of study participants

\begin{tabular}{lc}
\hline \multicolumn{1}{c}{ Characteristic } & Data \\
\hline TST (minutes) & $395.3 \pm 55.2$ \\
Arousal index (N/h) & $13.7 \pm 7.2$ \\
Stage N1 (\% TST) & $9.7 \pm 5.3$ \\
Stage N2 (\% TST) & $51.3 \pm 8.1$ \\
Stage N3 (\% TST) & $21.4 \pm 6.3$ \\
Stage R (\% TST) & $17.5 \pm 4.2$ \\
Sleep efficiency (\%) & $86.2 \pm 11.6$ \\
Sleep latency (minutes) & $14.5 \pm 20.6$ \\
REM latency (minutes) & $142.8 \pm 68.5$
\end{tabular}

Data were presented as mean \pm standard deviation. TST: total sleep time, N1: stage 1 sleep, N2: stage 2 sleep, N3: stage 3 sleep, R: stage rapid eye movement sleep, REM: rapid eye movement with both scoring rules. The specialists were blinded to patients' medical information. Manual scoring was performed epoch by epoch according to both pediatric and adult guidelines recommended in the 2007 and 2012 AASM scoring manuals (Table 1). When we scored the PSG results based on the adult guidelines in the 2007 manual, we used the hypopnea rule A. AHI was defined as the sum of apneas and hypopneas divided by total sleep time. The cut-off values of AHI for diagnosing sleep apnea in pediatric and in adult guidelines were defined as $\geq 1$ event per hour of sleep and as $\geq 5$ events per hour of sleep, respectively. ${ }^{11-13)}$

\section{Statistical analysis}

Statistical analyses were performed using SPSS software (ver. 19.0; IBM Corp., Armonk, NY, USA) and MedCalc (ver. 11.2; MedCalc Software, Mariakerke, Belgium). Statistical significance was considered when $p$ value was less than 0.05 . Data were expressed as mean and standard deviation for continuous variables or numbers and percentages for frequency variables. Bland-Altman plot, paired t-test, McNemar test, chi-squared test, and kappa statistics were used to characterize each variable accordingly.

\section{Results}

\section{Study participants}

A total of 106 Korean adolescents (aged 13 to 18) years were enrolled in this study. The characteristics of the participants are summarized in Table 2. Sleep architecture of participants is shown in Table 3.

\section{Polysomnography results}

Results of overnight PSG according to 2007 and 2012 meth-

Table 4. Polysomnographic results of study subjects

\begin{tabular}{|c|c|c|c|c|}
\hline & \multicolumn{2}{|c|}{2007 AASM scoring manual } & \multicolumn{2}{|c|}{2012 AASM scoring manual } \\
\hline & Pediatric score & Adult score & Pediatric score & Adult score \\
\hline $\mathrm{AHI}(\mathrm{N} / \mathrm{h})$ & $5.15 \pm 9.30$ & $3.66 \pm 8.57$ & $5.85 \pm 9.33$ & $5.76 \pm 9.31$ \\
\hline $\mathrm{OAI}(\mathrm{N} / \mathrm{h})$ & $1.21 \pm 4.47$ & $1.18 \pm 4.45$ & $1.21 \pm 4.46$ & $1.18 \pm 4.45$ \\
\hline $\mathrm{CAI}(\mathrm{N} / \mathrm{h})$ & $0.68 \pm 0.84$ & $0.61 \pm 0.81$ & $0.68 \pm 0.83$ & $0.60 \pm 0.81$ \\
\hline $\mathrm{MAI}(\mathrm{N} / \mathrm{h})$ & $0.20 \pm 1.13$ & $0.20 \pm 1.13$ & $0.20 \pm 1.13$ & $0.20 \pm 1.13$ \\
\hline $\mathrm{HI}(\mathrm{N} / \mathrm{h})$ & $3.06 \pm 5.38$ & $1.68 \pm 4.43$ & $3.74 \pm 5.58$ & $3.76 \pm 5.59$ \\
\hline RERA (N/h) & $3.50 \pm 3.07$ & $4.24 \pm 3.46$ & $2.95 \pm 2.79$ & $2.95 \pm 2.78$ \\
\hline $\mathrm{RDI}(\mathrm{N} / \mathrm{h})$ & $8.66 \pm 10.13$ & $7.91 \pm 9.68$ & $8.79 \pm 10.07$ & $8.71 \pm 10.03$ \\
\hline ODI (N/h) & $4.27 \pm 8.02$ & $3.12 \pm 7.16$ & $4.32 \pm 8.01$ & $4.31 \pm 8.01$ \\
\hline
\end{tabular}

Data were presented as mean \pm standard deviation. AASM: American Academy of Sleep Medicine, AHI: apnea hypopnea index, OAl: obstructive apnea index, CAl: central apnea index, MAl: mixed apnea index, HI: Hypopnea index, RERA: respiratory effort-related arousal, RDI: respiratory disturbance index, ODI: oxygen desaturation index 
od are summarized in Table 4. When AHI was calculated according to both 2007 and 2012 methods, the pediatric scores were significantly $(p<0.001)$ higher than the adult scores (Table 5). There were significantly $(p<0.001)$ higher scores of pediatric $\mathrm{AHI}_{2012}$ (AHI using 2012 AASM scoring manual) compared with the scores of pediatric $\mathrm{AHI}_{2007}$ (AHI using 2007 AASM scoring manual). Adult $\mathrm{AHI}_{2012}$ was significantly $(p<0.001)$ higher than adult $\mathrm{AHI}_{2007}$. The difference between pediatric $\mathrm{AHI}_{2012}$ and adult $\mathrm{AHI}_{2012}$ was significantly $(p<$ 0.001) less than the difference between pediatric $\mathrm{AHI}_{2007}$ and adult $\mathrm{AHI}_{2007}$ scores (Table 6).

\section{Agreement between pediatric and adult rules in 2007 and 2012 AASM scoring manuals}

Bland-Altman plots were performed to analyze the agreement between pediatric and adult guidelines in 2007 and 2012 recommendations (Fig. 1). The mean difference between pediatric $\mathrm{AHI}_{2007}$ and adult $\mathrm{AHI}_{2007}$ guidelines was 1.5. The limits of agreement ranged from -1.7 to 4.7 (Fig. 1A). The

Table 5. Comparison between pediatric $\mathrm{AHI}$ and adult $\mathrm{AHI}$ according to 2007 and 2012 AASM scoring manuals

\begin{tabular}{lccc}
\hline & Pediatric rule & Adult rule & p-value \\
\hline $\mathrm{AH}_{2007}$ & $5.15 \pm 9.30$ & $3.66 \pm 8.57$ & $<0.001$ \\
$\mathrm{AHI}_{2012}$ & $5.85 \pm 9.33$ & $5.76 \pm 9.31$ & $<0.001$ \\
p-value & $<0.001$ & $<0.001$ & \\
\hline
\end{tabular}

Data were presented as mean \pm standard deviation. $\mathrm{AH}_{2007}$ : $\mathrm{AHI}$ using 2007 AASM scoring manual, $\mathrm{AH}_{2012}$ : AHI using 2012 AASM scoring manual, AHI: apnea hypopnea index, AASM: American Academy of Sleep Medicine

Table 6. Comparison for difference between pediatric $\mathrm{AHI}$ and adult AHI according to 2007 and 2012 AASM scoring manuals

\begin{tabular}{ccc}
\hline $\begin{array}{c}\text { Pediatric } \mathrm{AHH}_{2007}- \\
\text { adult } \mathrm{AH}_{2007}\end{array}$ & $\begin{array}{c}\text { Pediatric } \mathrm{AH}_{2012}- \\
\text { adult } \mathrm{AH}_{2012}\end{array}$ & p-value \\
\hline $1.49 \pm 1.64$ & $0.08 \pm 0.14$ & $<0.001$ \\
\hline
\end{tabular}

Data were presented as mean \pm standard deviation. $\mathrm{AH}_{2007}$ : $\mathrm{AHI}$ using 2007 AASM scoring manual, $\mathrm{AH}_{2012}$ : AHI using 2012 AASM scoring manual, AHI: apnea hypopnea index, AASM: American Academy of Sleep Medicine mean difference between pediatric $\mathrm{AHI}_{2012}$ and adult $\mathrm{AHI}_{2012}$ was 0.08 . The limits of agreement ranged from -0.20 to 0.36 (Fig. 1B).

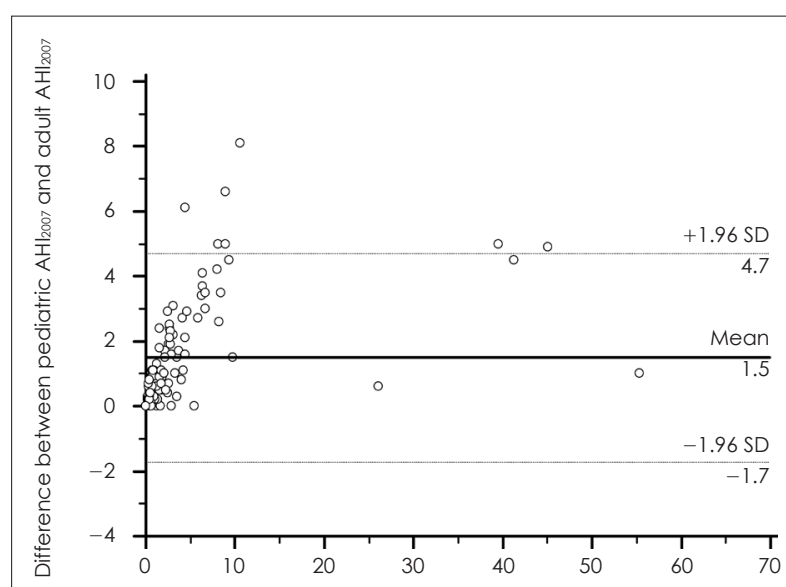

A Mean of pediatric $\mathrm{AH}_{2007}$ and adult $\mathrm{AH}_{2007}$

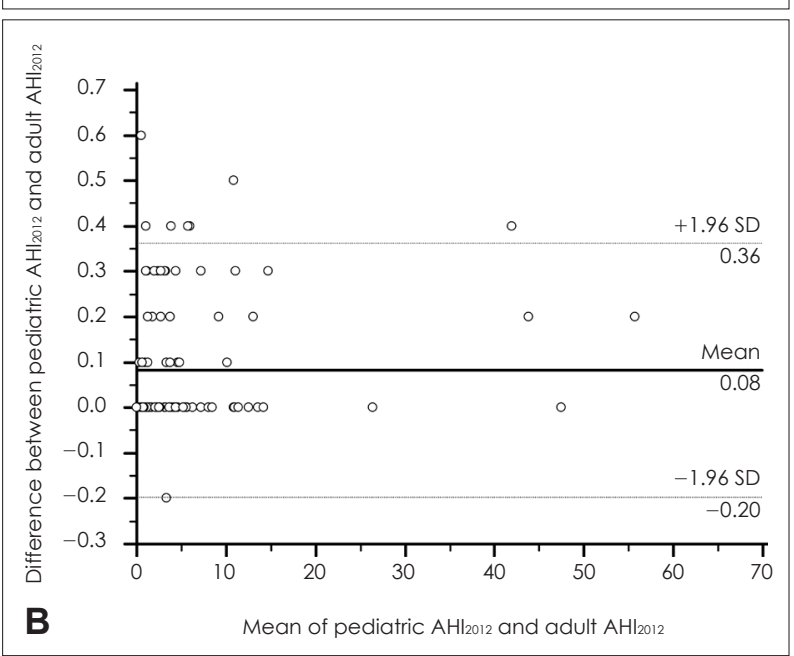

Fig. 1. Bland-Altman plots for 2007 (A) and 2012 (B). AASM scoring manual. $\mathrm{AHI}_{2007}$ : AHI using 2007 AASM scoring manual; $\mathrm{AHI}_{2012}$ : AHI using 2012 AASM scoring manual; solid line represents the mean difference; dotted line suggests $\pm 1.96 \mathrm{SD}$. AASM: American Academy of Sleep Medicine, AHI: apnea hypopnea index, SD: standard deviation.

Table 7. McNemar tests for pediatric and adult rule in 2007 and 2012 AASM scoring manuals

\begin{tabular}{|c|c|c|c|}
\hline & Sleep apnea using adult rule $(+)$ & Sleep apnea using adult rule $(-)$ & Total $(n, \%)$ \\
\hline \multicolumn{4}{|l|}{2007 AASM scoring manual } \\
\hline Sleep apnea using pediatric rule $(+)$ & 16 & 59 & $75(70.8)$ \\
\hline Sleep apnea using pediatric rule (-) & 0 & 31 & $31(29.2)$ \\
\hline Total $(n, \%)$ & $16(15.1)$ & $90(84.9)$ & 106 \\
\hline \multicolumn{4}{|l|}{2012 AASM scoring manual } \\
\hline Sleep apnea using pediatric rule $(+)$ & 33 & 51 & $84(79.2)$ \\
\hline Sleep apnea using pediatric rule (-) & 0 & 22 & $22(20.8)$ \\
\hline Total $(n, \%)$ & $33(31.1)$ & $73(68.9)$ & 106 \\
\hline
\end{tabular}

AASM: American Academy of Sleep Medicine 


\section{Diagnostic discordance for adolescent sleep apnea}

Results of McNemar tests comparing pediatric and adult rules according to the 2007 and 2012 methods are summarized in Table 7 . A total of 75 subjects $(70.8 \%)$ were diagnosed with sleep apnea according to the pediatric guidelines in 2007 AASM scoring system. A total of 84 (79.2\%) were diagnosed with sleep apnea according to pediatric guidelines in the 2012 system. Sixteen subjects (15.1\%) were diagnosed with sleep apnea according to adult guidelines in the 2007 scoring recommendations for adult patients, whereas 33 subjects (31.1\%) were diagnosed with sleep apnea according to adult rules in the 2012 method.

\section{Discussion}

We evaluated the differences in scoring with overnight PSG using both 2007 and 2012 AASM scoring manuals when pediatric and adult scoring guidelines were adopted for adolescents for the diagnosis of sleep apnea. This study showed significant differences among AHIs calculated according to the AASM guidelines (2007 and 2012 for the diagnosis of sleep apnea) in adolescents using overnight PSG. Pediatric $\mathrm{AHI}_{2012}$ was higher than pediatric $\mathrm{AHI}_{2007}$. Adult $\mathrm{AHI}_{2012}$ was also significantly higher than adult $\mathrm{AHI}_{2007}$ in this study. $\mathrm{Ba}$ Hammam, et al. ${ }^{14)}$ reported that $\mathrm{AHI}_{2012}$ was higher than $\mathrm{AHI}_{2007}$ in adults. These differences were mainly attributed to altered hypopnea definition for oxygen desaturation (from $\geq 3 \%$ or $\geq 4 \%$ to $\geq 3 \%$ desaturation from pre-event baseline) in the 2012 recommendations. No study has compared the difference between pediatric $\mathrm{AHI}_{2007}$ and $\mathrm{AHI}_{2012}$. Higher pediatric $\mathrm{AHI}_{2012}$ compared with pediatric $\mathrm{AHI}_{2007}$ in this study might be ascribed to revised hypopnea criteria for peak signal excursion drop (from $\geq 50 \%$ to $\geq 30 \%$ ).

Even though PSG is the most important diagnostic tool for sleep apnea, there is no scoring method for PSG for diagnosis of sleep apnea specifically in adolescents. Scoring guidelines for adolescents were based on both pediatric and adult rules in the 2007 and 2012 recommendations. A few studies investigating adolescent sleep apnea have reported higher pediatric $\mathrm{AHI}_{2007}$ than adult $\mathrm{AHI}_{2007}{ }^{9,15)}$ which is similar to our study results. Our results revealed that the difference between pediatric $\mathrm{AHI}_{2012}$ and adult $\mathrm{AHI}_{2012}$ was much less than the difference between pediatric $\mathrm{AHI}_{2007}$ and adult $\mathrm{AHI}_{2007}$. Bland-Altman plot showed that the mean difference between pediatric $\mathrm{AHI}_{2012}$ and adult $\mathrm{AHI}_{2012}$ decreased from 1.5 to 0.08 compared with the mean difference between pe- diatric $\mathrm{AHI}_{2007}$ and adult $\mathrm{AHI}_{2007}$.

According to the pediatric hypopnea criteria in the 2012 system, the revised definition for peak signal excursions (30\% decline compared with pre-event baseline) was similar to the adult hypopnea criteria in 2012 method. This change was probably the key factor that markedly decreased the difference between pediatric and adult $\mathrm{AHI}_{2012}$. Our results support the contention that the 2012 AASM manual resulted in fewer differences in AHI between pediatric and adult recommendations compared with the 2007 recommendations in diagnosing sleep apnea among adolescents. ${ }^{10)}$ Despite a marked decrease in differences between pediatric and adult AHI scores under the 2012 method compared with earlier method, our results showed that pediatric $\mathrm{AHI}_{2012}$ was still slightly higher than adult $\mathrm{AHI}_{2012}$. However, the difference between pediatric $\mathrm{AHI}_{2012}$ and adult $\mathrm{AHI}_{2012}$ was only $0.08 \pm 0.14$. We believe that this small difference of AHI between mean pediatric and adult AHIs was not clinically significant in diagnosing sleep apnea in adolescents. Therefore, both pediatric and adult guidelines can be used for the diagnosis of adolescent sleep apnea using the 2012 method without significant limitations compared with the 2007 method.

There is a significant discordance in diagnosis for adolescent sleep apnea between pediatric and adult rules in both 2007 and 2012 methods. Comparison of the 2012 and 2007 recommendations revealed an increase in the rate of positive diagnosis from $70.8 \%$ to $79.2 \%$ in pediatric guidelines, and from $15.1 \%$ to $31.1 \%$ in adult guidelines (Table 7 ). The rate of diagnostic discordance of sleep apnea in adolescents between pediatric and adult guidelines in the 2007 recommendations was $55.7 \%$ [ $95 \%$ confidence interval (CI): $15.1 \%$ to $70.8 \%$ ]. On the other hand, the discordant rate between the two systems decreased to $48.1 \%$ (95\% CI: $31.1 \%$ to $79.2 \%$ ) under the 2012 method, suggesting improvement without any statistical significance between the two discordant rates $(p=0.332)$. Overall, the results indicate poor agreement between pediatric and adult rules in both 2007 and 2012 methods for diagnosis of adolescent sleep apnea [kappa $=0.137$ (95\% CI: 0.063 to 0.211 ) and 0.212 (95\% CI: 0.116 to 0.308$)$ respectively].

For the diagnosis of sleep apnea, the commonly used cutoff value of AHI for pediatric patients is $\geq f$ and $\geq 5$ for adult patients. $^{11,12)}$ Because of these different cut-off values, the diagnostic discordance among these rules may occur even when pediatric AHI is similar to adult AHI. Although adolescents are physically more similar to adults than children, their growth and development are not fully complete. Sleep problems in 
adolescence may have a deleterious effect on physical and mental health: behavioral problems, poor academic achievement, and impaired emotional regulation. ${ }^{16)}$ Because adolescence is a dynamic transition period from childhood to adulthood, adolescents share a few common features with children or adults in sleep-related breathing disorder. In adolescents, obstructive apneas rarely last more than $10 \mathrm{~s}$. Most obstructive events do not terminate in a respiratory arousal as in younger children. ${ }^{17,18)}$ Adenotonsillar hypertrophy is an important risk factor in adolescent OSA. However, obesity is a more important risk factor than adenotonsillar hypertrophy in adolescent OSA owing to the increased prevalence of obesity. ${ }^{19-21)}$ There is no sexual difference in prevalence of OSA in younger children. However, in adolescents, the prevalence of OSA is higher in boys than in girls as in adults. ${ }^{22,23)}$ The respiratory rate of adolescents during sleep is slower than in early childhood. It is similar to the respiratory rate of middle-aged adults, ${ }^{15,24)}$ and consistent with our results.

Various features of adolescent sleep apnea complicate the diagnosis of adolescent sleep apnea. There are many studies for children and adult in sleep apnea. However, studies for adolescents are limited. The pathophysiology of adolescent sleep apnea has not been completely established. Currently, there is no specific scoring method for PSG in diagnosing sleep apnea in adolescents. Diagnosis and management of adolescent sleep apnea are difficult and challenging problems. Our results showed that even if pediatric hypopnea rules are revised similar to adult rules in the 2012 recommendations, differences in sleep apnea diagnosis in adolescents are still apparent under pediatric or adult guidelines. The different cut-off values of AHI for diagnosis of sleep apnea using the two scoring guidelines may be the primary factor. Both cut-off values of AHI are clinically significant. However, it is important to determine the cut-off value of AHI that is more reasonable for the diagnosis of sleep apnea in adolescents. Further studies and consensus of study groups are needed.

This study has a few limitations. We evaluated polysomnographic results. However, we did not evaluate other important factors for sleep apnea in adolescents such as developmental aspects, especially Tanner stages. In addition, we studied Korean adolescents in a single center. We did not evaluate the differences in other racial or ethnic populations or countries. The number of female subjects was only 31. Data from female subjects were limited compared with data derived from males.

In conclusion, the differences in AHI scores between pedi- atric and adults under the 2012 AASM scoring recommendations were substantially lower than in the 2007 AASM scoring guidelines. Both pediatric and adult guidelines in the 2012 manual are appropriate for the diagnosis of adolescent sleep apnea without significant challenges compared with the 2007 recommendations. However, the pediatric guideline is still associated with a higher rate of diagnosis for sleep apnea in adolescents than the adult guideline in 2012 recommendations. Different cut-off values for diagnosing sleep apnea between pediatric and adult systems may contribute to the discrepancy. Further studies are needed to decide the cut-off value that is more reasonable for diagnosis of sleep apnea in adolescents.

\section{Acknowledgments}

This was not an industry-supported study. This study was supported by a grant of the Korean Health Technology R\&D Project, Ministry of Health \& Welfare, Republic of Korea (HC15C3415). The funders had no role in study design, data collection and analysis, decision to publish, or preparation of the manuscript.

\section{REFERENCES}

1) Loughlin GM, Brouillette RT, Brooke LJ, Carroll JL, Chipps BE, England SJ, et al. Standards and indications for cardiopulmonary sleep studies in children. American Thoracic Society. Am J Respir Crit Care Med 1996;153(2):866-78.

2) Marcus CL, Brooks LJ, Draper KA, Gozal D, Halbower AC, Jones $\mathrm{J}$, et al. Diagnosis and management of childhood obstructive sleep apnea syndrome. Pediatrics 2012;130(3):576-84.

3) Perez-Chada D, Perez-Lloret S, Videla AJ, Cardinali D, Bergna MA, Fernández-Acquier M, et al. Sleep disordered breathing and daytime sleepiness are associated with poor academic performance in teenagers. A study using the Pediatric Daytime Sleepiness Scale (PDSS). Sleep 2007;30(12):1698-703.

4) Bass JL, Corwin M, Gozal D, Moore C, Nishida H, Parker S, et al. The effect of chronic or intermittent hypoxia on cognition in childhood: a review of the evidence. Pediatrics 2004;114(3):805-16.

5) Section on Pediatric Pulmonology, Subcommittee on Obstructive Sleep Apnea Syndrome. American Academy of Pediatrics. Clinical practice guideline: diagnosis and management of childhood obstructive sleep apnea syndrome. Pediatrics 2002;109(4):704-12.

6) Iber C, Ancoli-Israel S, Chesson A, Quan SF. The AASM manual for the scoring of sleep and associated events: rules, terminology and technical specifications. Westchester: American Academy of Sleep Medicine;2007.

7) Marcus CL. Sleep-disordered breathing in children. Am J Respir Crit Care Med 2001;164(1):16-30.

8) Sisk CL, Foster DL. The neural basis of puberty and adolescence. Nat Neurosci 2004;7(10):1040-7.

9) Accardo JA, Shults J, Leonard MB, Traylor J, Marcus CL. Differences in overnight polysomnography scores using the adult and pediatric criteria for respiratory events in adolescents. Sleep 2010;33(10): 1333-9.

10) Berry RB, Budhiraja R, Gottlieb DJ, Gozal D, Iber C, Kapur VK, et al. Rules for scoring respiratory events in sleep: update of the 2007 AASM Manual for the Scoring of Sleep and Associated Events. Deliberations of the Sleep Apnea Definitions Task Force of the American Academy of Sleep Medicine. J Clin Sleep Med 2012;8(5): 
597-619.

11) Don DM, Geller KA, Koempel JA, Ward SD. Age specific differences in pediatric obstructive sleep apnea. Int J Pediatr Otorhinolaryngol 2009;73(7):1025-8

12) Mannarino MR, Di Filippo F, Pirro M. Obstructive sleep apnea syndrome. Eur J Intern Med 2012;23(7):586-93.

13) American Academy of Sleep Medicine. International classification of sleep disorders: diagnostic \& coding manual. Westchester, Illinois: American Academy of Sleep Medicine;2005.

14) BaHammam AS, Obeidat A, Barataman $K$, Bahammam SA, Olaish AH, Sharif MM. A comparison between the AASM 2012 and 2007 definitions for detecting hypopnea. Sleep Breath 2014;18(4):767-73.

15) Tapia IE, Karamessinis L, Bandla P, Huang J, Kelly A, Pepe M, et al. Polysomnographic values in children undergoing puberty: pediatric vs. adult respiratory rules in adolescents. Sleep 2008;31(12):1737-44.

16) Touitou Y. Adolescent sleep misalignment: a chronic jet lag and a matter of public health. J Physiol Paris 2013;107(4):323-6.

17) Marcus CL, Omlin KJ, Basinki DJ, Bailey SL, Rachal AB, Von Pechmann WS, et al. Normal polysomnographic values for children and adolescents. Am Rev Respir Dis 1992;146(5 Pt 1):1235-9.

18) Fuentes-Pradera MA, Botebol G, Sánchez-Armengol A, Carmona
C, García-Fernández A, Castillo-Gómez J, et al. Effect of snoring and obstructive respiratory events on sleep architecture in adolescents. Arch Pediatr Adolesc Med 2003;157(7):649-54

19) Kelsey MM, Zaepfel A, Bjornstad P, Nadeau KJ. Age-related consequences of childhood obesity. Gerontology 2014;60(3):222-8.

20) Huang J, Pinto SJ, Yuan H, Katz ES, Karamessinis LR, Bradford RM, et al. Upper airway collapsibility and genioglossus activity in adolescents during sleep. Sleep 2012;35(10):1345-52.

21) Redline S, Storfer-Isser A, Rosen CL, Johnson NL, Kirchner HL, Emancipator J, et al. Association between metabolic syndrome and sleep-disordered breathing in adolescents. Am J Respir Crit Care Med 2007;176(4):401-8.

22) Lumeng JC, Chervin RD. Epidemiology of pediatric obstructive sleep apnea. Proc Am Thorac Soc 2008;5(2):242-52.

23) Sánchez-Armengol A, Ruiz-García A, Carmona-Bernal C, BotebolBenhamou G, García-Díaz E, Polo-Padillo J, et al. Clinical and polygraphic evolution of sleep-related breathing disorders in adolescents. Eur Respir J 2008;32(4):1016-22.

24) Krieger J, Maglasiu N, Sforza E, Kurtz D. Breathing during sleep in normal middle-aged subjects. Sleep 1990;13(2):143-54. 